\section{Vers un ciblage du TGF- $\beta$ pour de futures immunothérapies anti-cancéreuses plus efficaces}

Rayan Foughali ${ }^{1}$, Julien C. Marie ${ }^{2}$
${ }^{1}$ Master de cancérologie, ISPB, Université Claude Bernard Lyon 1, Lyon 69008, France.

${ }^{2}$ Tumor escape resistance immunity

department, Cancer research center of Lyon, Lyon 69008, France.

rayan.foughali@etu.univ-lyonl.fr julien.marie@inserm.fr

Au sein de la masse tumorale, le TGF- $\beta$ est produit par les cellules tumorales et par des cellules de leur microenvironnement. Si le TGF- $\beta$ produit directement par la cellule tumorale semble être important dans la répression des lymphocytes T effecteurs [8], les cellules tumorales recrutent notamment des fibroblastes, appelés aussi fibroblastes associés au cancer (cancer-associatedfibroblasts, CAF) qui, par leur sécrétion de TGF- $\beta$, favoriseraient aussi un microenvironnement tumoral immunosuppresseur [7]. Le TGF- $\beta$ produit par les CAF contribue également à l'exclusion des lymphocytes $T$ hors du microenvironnement tumoral, et est donc un frein à l'action des immunothérapies ciblant ces lymphocytes [7].

Cibler le TGF- $\beta$ : effets potentiellement bénéfiques $d$ 'inhibiteurs

L'utilisation de galunisertib chez la souris augmente l'activation des lymphocytes T et réduit le nombre de métastases

Compte tenu du puissant rôle immunosuppresseur du TGF- $\beta$ sur les lymphocytes $T$, plusieurs équipes de recherche ont testé des inhibiteurs de la signalisation induite par le TGF- $\beta$, voire des neutralisateurs de ce polypeptide. Les travaux récents de Tauriello et al. révèlent que le traitement de souris par le galunisertib, un inhibiteur du récepteur $d u$ TGF- $\beta$, augmente l'activation des lymphocytes T effecteurs dans la tumeur, et est suffisant pour réduire le nombre de métastases du cancer du côlon au foie, sans toutefois prolonger la survie des animaux [7]. 


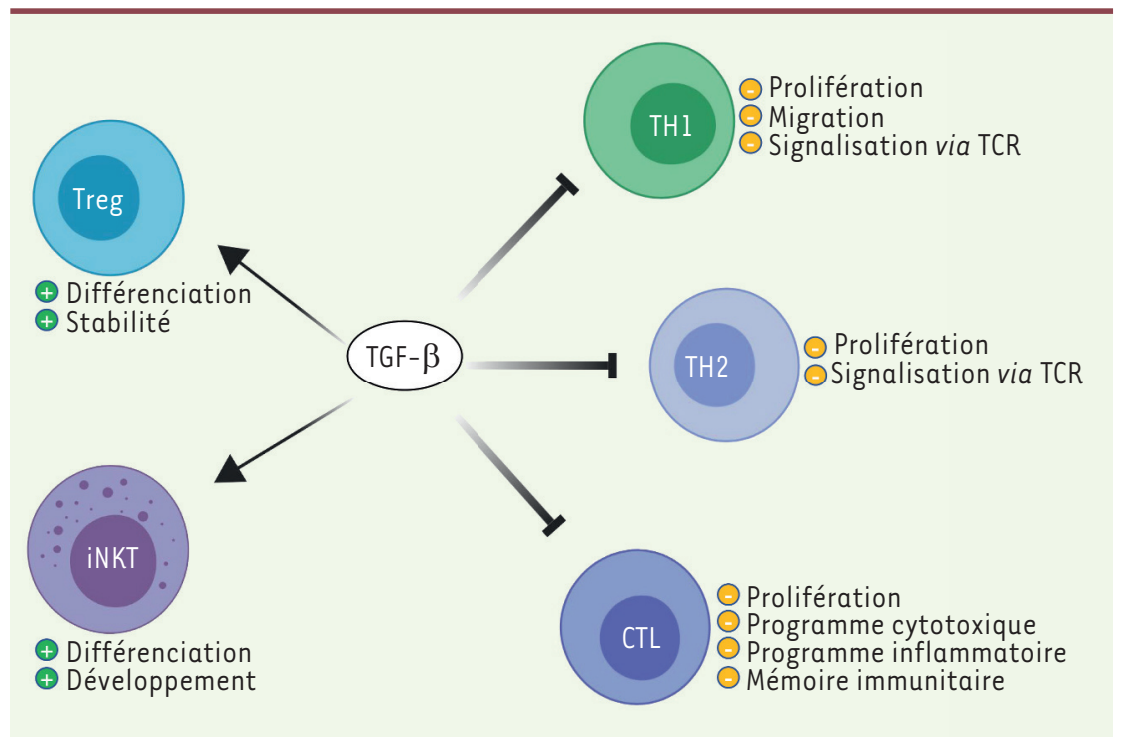

Figure 1. Le TGF- $\beta$ entraîne une diminution des processus inflammatoires et une immunotolérance via une activation de lymphocytes régulateurs tels que les lymphocytes T régulateurs (Treg) et les innate-like T cells (iNKT) (bien que ceux-ci puissent avoir des effets pro-inflammatoires et antitumoraux) et une inhibition de la prolifération et des fonctions effectrices des lymphocytes T effecteurs tels que les lymphocytes $T$ helper 1 et 2 ( $T H 1, T H 2)$ et lymphocytes $T$ cytotoxiques $C D 8^{+}$(CTL).

La combinaison d'immunothérapies antiPD1 et anti-TGF- $\beta$ permet une meilleure survie

Les conclusions de deux études récentes s'accordent sur le fait que, chez l'homme comme chez la souris, les tumeurs avec une forte production de TGF- $\beta$ sont beaucoup moins sensibles aux immunothérapies par anticorps anti-PD-1 ou antiPDL-1. En effet, une analyse de tumeurs métastatiques urothéliales après une immunothérapie anti-PDLl montre que les patients qui ne répondent pas ou peu au traitement présentent une forte signature génique de l'activation de la signalisation par le TGF- $\beta$ dans les lymphocytes T [9]. De même, chez la souris, les tumeurs colorectales ou les carcinomes mammaires exprimant des taux élevés de TGF- $\beta$ sont réfractaires aux traitements anti-PD-1 ou anti-PDL-1 [7,9]. Remarquablement, l'association d'un traitement par un inhibiteur de la signalisation du TGF- $\beta$ et d'une immunothérapie anti-PD-1 augmente très nettement la survie des animaux [7]. Si les auteurs de ces deux travaux proposent que le TGF- $\beta$, par sa capacité à réprimer l'infiltrat lymphocy- de TGF- $\beta$, cet acteur majeur de la réponse immunitaire, pourrait cependant entraîner des effets indésirables graves d'autoimmunité; dans cette même étude, une exposition chronique de plus de 3 mois au galunisertib entraîne une toxicité touchant de multiples organes [10].

\section{Le futur du TGF- $\beta$ dans la médecine personnalisée}

II a été montré par Ozdemir et al. [11] que la génération de souris transgéniques dépourvues de myofibroblastes dans l'adénocarcinome ductal pancréatique aboutit à des tumeurs invasives et non-différenciées, ayant des propriétés hypoxiques augmentées, un rapport lymphocytes T effecteurs / T régulateurs diminué, et une augmentation d'expression de la protéine CTLA-4, ce qui réduit considérablement la survie de ces animaux. Ces résultats, corroborés par une analyse chez des patients atteints d'adénocarcinome ductal pancréatique et ne présentant que peu de myofibroblastes dans leur tumeur, prouvent que cibler les CAF, qui sont les principaux sécréteurs du TGF- $\beta$ dans ce type de cancer, ne représente pas une cible thérapeutique intéressante. De plus, il a été confirmé par plusieurs équipes qu'une stratégie ciblant le TGF- $\beta$ seul est peu efficace $[7,9]$. En revanche, l'inhibition du TGF- $\beta$ en combinaison avec d'autres thérapies pourrait représenter une stratégie efficace contre différents types de cancers. Une médecine personnalisée s'appuyant sur l'immunothérapie serait certes coûteuse, mais pourrait être la clé pour traiter efficacement certaines tumeurs. Grâce notamment à l'essor de techniques de séquençage à haut débit permettant de caractériser finement le profil moléculaire des tumeurs, la médecine personnalisée en oncologie s'est beaucoup développée ces dernières années. Elle est même devenue un standard de procédure dans un nombre croissant de cancers : les altérations telles que l'amplification de ERBB2 (HER2) dans le cancer du sein, les mutations de l'EGFR ou de KRAS/BRAF, respectivement dans le cancer du poumon à petites-cellules et les cancers colo-rectaux, ou encore 

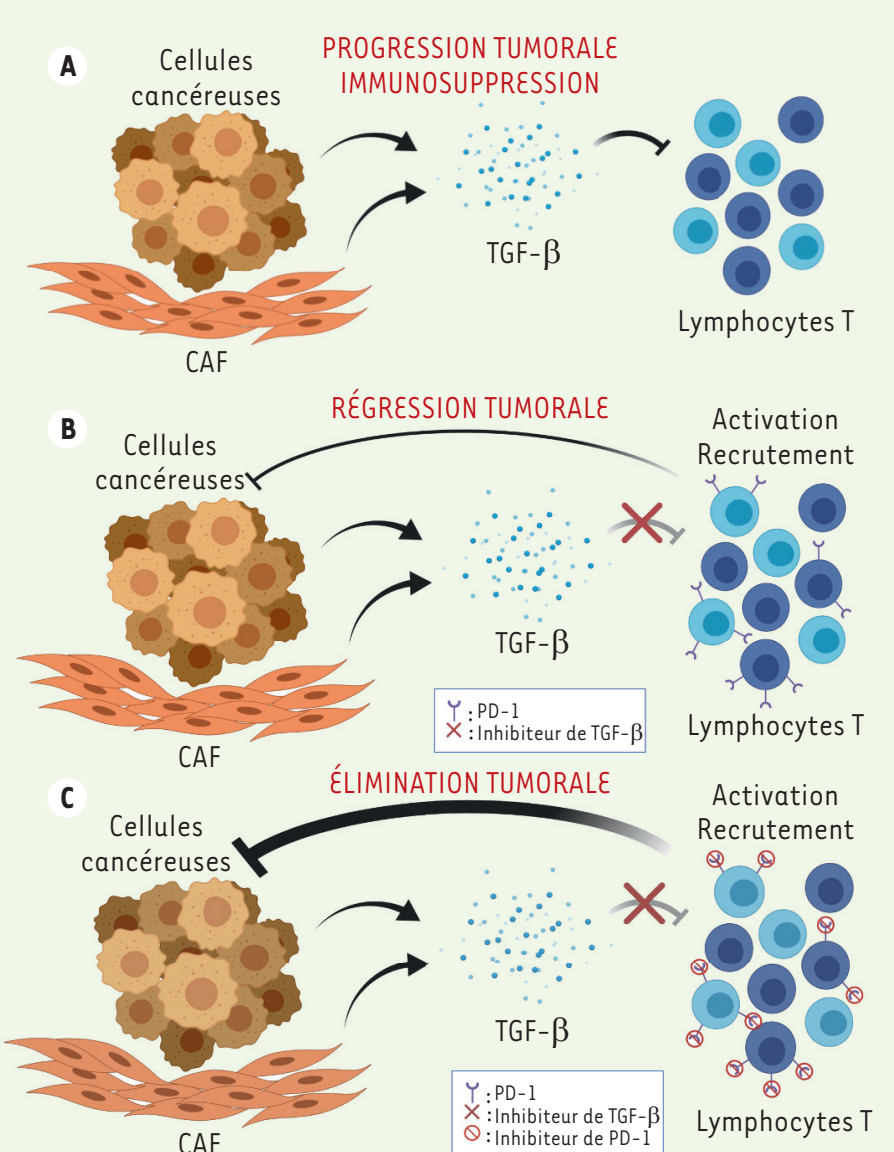

Figure 2. Schématisation de possibles immunothérapies contrant l'immunosuppression induite par la production de TGF- $\beta$ par la tumeur. A. Les cellules cancéreuses ainsi que les fibroblastes associés au cancer (CAF) sécrètent du TGF- $\beta$ en grande quantité. Sans traitement, les effets immunosuppresseurs du TGF- $\beta$ permettent à la tumeur de se développer et de progresser.

B. Avec un inhibiteur de la signalisation du TGF- $\beta$ tel que le galunisertib, la réponse immunitaire anti-tumorale se met en place par le biais des lymphocytes $T$, entraînant une régression de la masse tumorale. Les LT activés expriment la molécule PD-1 qui, en se liant à ses ligands PD-L1/ $P D-L 2$, réduit leur activité inflammatoire: la réponse anti-tumorale est partielle. $C$. Avec une combinaison d'inhibiteurs de TGF- $\beta$ et de PD-1, l'inhibition des lymphocytes T par PD-1 est levée, et la réponse anti-tumorale est complète.

les fusions BCR/ABL dans la leucémie myéloïde chronique, sont examinées de manière routinière.

L'analyse des signatures moléculaires de réponse des tumeurs aux immunothérapies constitue également un défi en oncologie pour les prochaines années, pour la recherche et pour le corps médical. Parmi ces immunothérapies, les inhibiteurs du TGF- $\beta$ pourraient jouer un rôle charnière [12]. Cependant, il faudrait que le TGF- $\beta$ ait été identifié préalablement comme un facteur clé du développement des optique, Bayin et al. ont d'aille de gliome de haut grade afin de tester la réponse patient-spécifique à une inhi- bition du TGF- $\beta$, ce modèle pouvant être étendu aux combinaisons d'inhibiteurs [13]. Le ciblage du TGF- $\beta$ pourrait également permettre d'affiner les immunothérapies qui se révèlent parfois inefficaces dans certains types de cancers, prouvant ainsi le potentiel de cette molécule dans la médecine personnalisée. $\diamond$ TGF- $\beta$-targeted immunotherapy aiming for new and efficient treatments of cancer

\section{LIENS D'INTÉRÊT}

Les auteurs déclarent n'avoir aucun lien d'intérêt concernant les données publiées dans cet article.

\section{RÉFÉRENCES}

1. Nishino M, Ramaiya N, Hatabu H, et al. Monitoring immune-checkpoint blockade: response evaluation and biomarker development. Nat Rev Clin Oncol 2017 ; $14: 655-8$.

2. Shull M, Ormsby I, Kier A, et al. Targeted disruption of the mouse transforming growth factor- $\beta 1$ gene results in multifocal inflammatory disease. Nature 1992 ; $359:$ : 693-9.

3. Li M, Sanjabi S, Flavell R. Transforming growth factor- $\beta$ controls development, homeostasis, and tolerance of $T$ cells by regulatory $T$ cell-dependent and -independent mechanisms. Immunity. 2006 ; $25:$ 455-71.

4. Marie J, Liggitt D, Rudensky A. Cellular mechanisms of fatal early-onset autoimmunity in mice with the T cell-specific targeting of transforming growth factor- $\beta$ receptor. Immunity $2006 ; 25: 441-54$.

5. Gorelik L, Flavell R. Immune-mediated eradication of tumors through the blockade of transforming growth factor- $\beta$ signaling in T cells. Nat Med 2001 ; 7 : 1118-22.

6. Sakaguchi S, Yamaguchi T, Nomura T, et al. Regulatory T cells and immune tolerance. Cell 2008 ; 133 : 775-87.

7. Tauriello DVF, Palomo-Ponce $S$, Stork D, et al. TGF $\beta$ drives immune evasion in genetically reconstituted colon cancer metastasis. Nature 2018 ; 554 : 538-43.

8. Courau T, Nehar-Belaid D, Florez L, et al. TGF- $\beta$ and VEGF cooperatively control the immunotolerant tumor environment and the efficacy of cancer immunotherapies. JCl Insight 2016; 1 : e85974.

9. Mariathasan S, Turley SJ, Nickles D, et al. TGF $\beta$ attenuates tumour response to PD-L1 blockade by contributing to exclusion of T cells. Nature 2018 ; $554: 544-8$.

10. Herbertz S, Sawyer JS, Stauber AJ, et al. Clinical development of galunisertib (LY2157299 monohydrate), a small molecule inhibitor of transforming growth factor-beta signaling pathway. Drug Des Dev Ther 2015 ; 9 : 4479-99.

11. Özdemir BC, Pentcheva-Hoang T, Carstens JL, et al. Depletion of carcinoma-associated fibroblasts and fibrosis induces immunosuppression and accelerates pancreas cancer with reduced survival. Cancer Cell $2014 ; 25: 719-34$.

12. Dahmani A, Delisle J. TGF- $\beta$ in T cell biology: implications for cancer immunotherapy. Cancers $2018 ; 10(6): 194$

13. Bayin N, Ma L, Thomas C, et al. Patient-specific screening using high-grade glioma explants to determine potential radiosensitization by a TGF- $\beta$ small molecule inhibitor. Neoplasia 2016 ; 18 : 795-805. 